\title{
The mechanism initiating and controlling monozygotic twinning in humans remains to be elucidated
}

Nine-banded armadillos almost invariably give birth to litters of monozygotic quadruplets (assuming no prenatal losses). The mechanism for this is thought to be related to delayed implantation following ovum fertilization. ${ }^{1-3}$ As a consequence of this evolutionary adaptation, offspring are born in the early spring (most commonly in March or April), rather than face the risks of birth in the winter months. Prior to implantation, the fertilised egg develops into a blastocyst vesicle bathed with endometrial fluids within the uterine cavity. During this delay, two consecutive divisions of growth centres occur, yielding four foetuses. (The mulita armadillo normally produces eight or more offspring from a single ovum.) Similarly, zygotic development, delayed artificially by temperature reduction or oxygen deprivation, experimentally enhances the rate of monozygotic twinning in selected test animals. ${ }^{4-6}$ The actual timing of implantation at the molecular level now seems to be regulated by blastocyst differentiation leading to trophoblastic elaboration of specific proteases and the process of integrin switching, ${ }^{7}$ as well as the activity of oncofoetal fibronectin. ${ }^{8}$

Therefore, it has been proposed that monozygotic twinning results from developmental arrest occurring very early in embryonic life, before the onset of tissue differentiation..$^{9,10}$ This may be a consequence of delayed implantation related to infrequent expression of restrained genetic capability or to biochemical malfunction. Recent examination of the seasonal variation of twin births tends to support this proposal. The report in the March 1999 issue of Twin Research points to the increased incidence of human twin deliveries in March and April. ${ }^{11}$ The length of gestation of twins is, within a couple of weeks, typically the same as singletons. As with other biological functions, the timing of successful fertilization (and hence delivery) especially in humans is the end result of the interaction of multiple factors including fecundity, diet, weather, economics, geography, and social issues. For example, a study of the seasonality of monozygotic twin birth rates in Great Britain was equivocal and inconclusive. ${ }^{12}$ Natural monozygotic multifoetal pregnancies are quite different from polyzygotic in aetiology, frequency, risk, and dependent influences (such as race and heredity). So too, in elucidating the mechanism of monozygotic twinning, it is important carefully to distinguish test subjects by zygosity.
Viable human quadruplets are, on average, born around 31-32 weeks of gestational life. ${ }^{13}$ In one registry of monozygotic quadruplets, delivery dates were reported in 10 of the 18 known sets in the world today. ${ }^{14}$ After adjusting for prematurity, the mean birth date is in April.

Thus, by analogy with the armadillo, these observations would be consistent with the proposal that twinning in humans is a reflection of evolutionary adaptations which have been largely suppressed or eliminated, but which reemerge from time to time (4 out of 1000 deliveries) in some contemporary pregnancies.

Dr Gary Steinman

Dept. of OBS/GYN, Long Island Jewish Medical Center, New Hyde Park, NY, 11040 USA

\section{References}

1 Hamlett GSW. The reproductive cycle in the armadillo. A Wiss Zool 1932; 141: 143.

2 McBee K, Baker RJ. Nine-banded armadillo. Mammalian Species, American Society of Mammalogists, vol. 162.

3 Nine-banded Armadillo, http://www.nsrl.ttu.edu/tmotl/ dasynove.htm.

4 Stockard CR. Developmental rate and structural expression. Am J Anat 1921; 28: 115.

5 Sturkie PD. The production of twins in Gallus domesticus. J Exp Zool 1946; 101: 51.

6 Newman $\mathrm{HH}$. The Physiology of Twinning, University of Chicago Press: Chicago, 1923.

7 Fernette PS, Wagner DD. Adhesion molecules, Part I. N Engl J Med 1996; 334: 1526.

8 Feinberg RF, Kliman $J$, Wang CL. Is oncofetal fibronectin a trophoblast glue for human implantation? Am J Pathol 1991; 138: 537.

9 Bulmer MG. The Biology of Twinning in Man, Clarendon: Oxford, 1970; pp 36-37.

10 Luke B, Keith LG. Monozygotic twinning as a congenital defect and congenital defects in monozygotic twins. Fetal Diagn Ther 1990; 5: 61-69.

11 Fellman J, Eriksson AW. Statistical analysis of the seasonal variation in the twinning rate. Twin Res 1999; 2: 22-29.

12 James WH. Seasonality in twin and triplet births. Ann Hum Biol 1980; 7: 163-175.

13 Lynch L, Berkowitz RL. The natural history of grand multifetal pregnancies and the effect of pregnancy reduction. In: Keith LG, Papiernik E, Keith DM, Luke B (eds). Multiple Pregnancy - Epidemiology, Gestation and Perinatal Outcome, Parthenon Publishing Group: 1995, pp 352-353.

14 Quadruplets, http://mypage.direct.ca/c/csamson/multiples .html. 